Sociologie et sociétés

\title{
Les aspirations des travailleuses de la garde d'enfants en milieu familial
}

\author{
À l'intersection de la trajectoire professionnelle, de la famille
} et de la migration

\section{Home day-care workers' aspirations At the intersection of professional career, family and migration}

\section{Martine D’Amours}

Volume 47, numéro 1, printemps 2015

Travail et informalité : nouvelles figures de l'exploitation et des mobilisations au nord et au sud

Work and the informal sector: new figures of exploitation and mobilisations in the north and south

URI : https://id.erudit.org/iderudit/1034422ar

DOI : https://doi.org/10.7202/1034422ar

Aller au sommaire du numéro

\section{Éditeur(s)}

Les Presses de l'Université de Montréal

\section{ISSN}

0038-030X (imprimé)

1492-1375 (numérique)

Découvrir la revue

\section{Citer cet article}

D’Amours, M. (2015). Les aspirations des travailleuses de la garde d'enfants en milieu familial : à l'intersection de la trajectoire professionnelle, de la famille et de la migration. Sociologie et sociétés, 47(1), 147-175.

https://doi.org/10.7202/1034422ar
Résumé de l'article

Le présent article porte sur une forme particulière de travail de soins, soit la garde d'enfants à domicile, telle qu'elle est exercée dans le cadre institutionnel québécois. Après avoir exposé la précarité des conditions d'emploi de celles qui exercent cette activité, formellement à titre de travailleuses indépendantes, il met l'accent sur l'appréciation subjective qu'elles en ont : quelle importance accordent-elles aux marges d'autonomie procurées par leur statut? Quelles voies privilégient-elles pour améliorer les conditions d'exercice de leur activité ? Comment envisagent-elles leur avenir professionnel ? Les résultats issus d'une enquête réalisée en 2011 auprès de 3769 responsables de services de garde en milieu familial révèlent une importante diversité à cet égard, expliquée par l'imbrication des inégalités construites dans la trajectoire professionnelle, de la division du travail au sein de la famille et de l'expérience de la migration. 


\title{
Les aspirations des travailleuses de la garde d'enfants en milieu familial
}

\author{
À l'intersection de la trajectoire professionnelle, \\ de la famille et de la migration
}

\section{MARTINE D'AMOURS}

Département des relations industrielles Université Laval

1025, avenue des Sciences-Humaines

Québec (Qc) Canada GıV oA6

Courriel: martine.damours@rlt.ulaval.ca

$\mathrm{L}$

A LITTÉRATURE SCIENTIFIQUE SUR LE TRAVAIL de soins révèle, au-delà de son fort taux de féminisation, la diversité du contenu et des contraintes de ces emplois (Avril et Cartier, 2014), de même que l'hétérogénéité des propriétés sociales des travailleuses qui les occupent (Devetter, 2012; Cartier et al., 2012; Avril, 2003, 2012). Nous inspirant d'une perspective intersectorielle, particulièrement intéressante en ce qu'elle permet de briser la représentation d'homogénéité au sein des catégories sociales dominées (Palomares et Testenoire, 2010), nous avons cherché à expliquer la diversité des aspirations professionnelles d'un groupe particulier de travailleuses de soins - les responsables de services de garde en milieu familial —, à l'intersection de plusieurs axes de structuration des inégalités.

Après avoir exposé la précarité des conditions d'emploi de celles qui exercent cette activité au Québec, formellement à titre de travailleuses indépendantes, l'article interroge l'appréciation subjective qu'elles en ont: quelle importance accordent-elles aux marges d'autonomie procurées par leur statut? Quelles voies privilégient-elles pour améliorer les conditions d'exercice de leur activité? Comment envisagent-elles leur avenir professionnel? Les résultats issus d'une enquête réalisée en 2011 auprès de 3769 responsables de services de garde en milieu familial révèlent une importante diversité à cet égard, expliquée par l'imbrication des inégalités construites dans la trajectoire 
professionnelle, de la division du travail au sein de la famille et de l'expérience de la migration.

\section{LA GARDE D'ENFANTS À DOMICILE AU QUÉBEC: UN EMPLOI «DU BAS DE L'ÉCHELLE »}

Au nombre de 15054 en 2013 (Gouvernement du Québec, 2015), les responsables de service de garde en milieu familial (RSG dans la suite du texte) accueillent les enfants dans leur domicile: au maximum 6 si elles sont seules et jusqu'à 9 si elles travaillent avec une assistante ${ }^{1}$.

Renversant les décisions des tribunaux qui leur avaient, au tournant des années 2000, attribué la qualification de salariées, le gouvernement du Québec les considère depuis 2003 comme des travailleuses autonomes ${ }^{2}$, mais cette autonomie est fortement contrainte. Selon l'analyse de Beaulieu (2011), depuis l'adoption de la politique familiale en $1997^{3}$, la majorité des éléments de l'organisation du travail de garde d'enfants à domicile sont en effet déterminés de l'extérieur, qu'il s'agisse du nombre d'heures d'ouverture ou du tarif. Il existe en outre un fort contrôle par les règles, qui se concrétise par des pratiques telles que les visites à l'improviste trois fois par année ou par l'obligation de remplir les fiches d'assiduité hebdomadaires comme condition du versement de la rémunération. Les marges d'autonomie résident dans le fait que les RSG peuvent décider des activités pédagogiques et conservent le pouvoir de choisir leur clientèle. Ce dernier élément revêt une grande importance aux yeux des RSG qui, parce qu'elles travaillent dans leur résidence, sont soucieuses de choisir des parents «compatibles» avec leurs valeurs et respectueux des règles de fonctionnement et de l'approche qu'elles privilégient (Beaulieu, 2011; D’Amours, 2013).

Établi par la loi et la réglementation, le principe de leur rémunération combine une subvention gouvernementale et une contribution parentale ${ }^{4}$. Le montant de la subvention (par jour et par enfant) est négocié collectivement depuis $2011^{5}$ et il inclut

1. Ce qui était le cas de $23 \%$ des RSG en mars 2012 (Gouvernement du Québec, 2014) et de 21,9\% dans notre échantillon.

2. Le terme «travail indépendant» est celui usité dans la littérature scientifique et dans la documentation statistique pour désigner l'indépendance juridique, qui n'est pas garante de l'autonomie dans l'exercice du travail. Mais comme les lois qui régissent l'activité (et les RSG elles-mêmes) utilisent le terme «travail autonome», nous utilisons indifféremment les deux appellations.

3. Avant 1997 en effet, les RSG (qui ne portent pas encore ce nom) apparaissent comme de véritables travailleuses indépendantes, qui déterminent les paramètres de leur activité (notamment les tarifs et les heures d'ouverture), tout en étant soumises à un contrôle visant à assurer le respect de normes de qualité (Lalonde-Graton, 2002), et assument les risques sociaux et professionnels associés au travail. À partir de 1997, plusieurs éléments de leur prestation de travail sont déterminés par la loi ou la réglementation.

4. Initialement fixée à $5 \$$, augmentée par la suite jusqu'à $7,30 \$$ par jour et par enfant, la contribution parentale est dorénavant (depuis avril 2015) modulée selon le revenu familial, variant entre 7,30 $\$$ et 20 \$ par jour et par enfant. Cette hausse de la contribution parentale pour une partie de la clientèle n'est pas destinée à bonifier les conditions de travail, mais plutôt à réduire les dépenses gouvernementales.

5. En vertu de la Loi sur la représentation de certaines personnes responsables d'un service de garde en milieu familial et sur le régime de négociation d'une entente collective les concernant, qui crée un régime 
le coût de la contribution à divers programmes sociaux: assurance collective, régime de retraite, indemnisation des lésions professionnelles, régime des rentes du Québec, assurance parentale, fonds des services de santé. Cette contribution du donneur d'ouvrage à la protection sociale fait de ce groupe (et de quelques autres, comme les artistes interprètes) une exception parmi les travailleurs indépendants, qui doivent assumer seuls le coût de telles protections, et qui, faute de moyens, s'en trouvent souvent dépourvus (Akyeampong et Sussman, 2003).

Bien que bonifiées par les négociations collectives, les conditions d'emploi des RSG demeurent inférieures à celles des éducatrices salariées des CPE. Le montant du financement est établi en considérant que la prestation de services d'une RSG équivaut à l'activité d'une éducatrice non qualifiée en CPE, à l'échelon 1 (commandant un salaire horaire de 15,39 \$ du $1^{\text {er }}$ avril 2014 au 31 mars 2015). Or $82 \%$ des éducatrices en garderie sont qualifiées ${ }^{6}$, et commandent, pour la même période, un salaire oscillant entre 17,42 \$ (échelon 1) et 23,09 \$ (échelon 10). Alors que les éducatrices en CPE (permanentes à temps complet) bénéficient en moyenne de 23,5 jours de congé payés ${ }^{7}$ et de 3 semaines de vacances après 2,7 années de service (4 semaines après 5,5 années) (Gouvernement du Québec, 2015), les RSG ont droit à 25 jours au titre des congés et des vacances, peu importe leurs états de service. Les RSG doivent offrir un service 10 heures par jour, en sus des heures consacrées aux tâches connexes (courses, confection de repas, lavage de jouets), ce qui excède largement la durée moyenne de la journée de travail en CPE.

Les RSG apparaissent en outre fragilisées par leur statut de travailleuses indépendantes, qui les exclut de la protection des lois d'ordre public, normes du travail et équité salariale notamment, ce qui amène Bernstein (2012) à conclure à une discrimination selon le genre ainsi qu'à des disparités importantes selon le statut d'emploi. Au total, les RSG apparaissent comme des indépendantes du bas de l'échelle ${ }^{8}$, évoluant dans une zone hybride caractérisée par une autonomie contrôlée et par certaines modalités de partage des risques associés au travail (Beaulieu 2011, Cox, 2005: 3; D’Amours, 2015).

\section{REVUE DE LA LitTÉRATURE SCIENTIFIQUE}

Au-delà de cette condition partagée par les RSG, la question des dimensions subjectives de leur rapport à l'emploi et à leur statut de travailleuse indépendante et, partant, de leurs aspirations professionnelles, demeure largement inexplorée. Trouvent-elles,

particulier permettant aux associations représentatives des RSG de négocier collectivement le montant de la subvention gouvernementale, et d'y inclure les frais relatifs à certains régimes de protection sociale, ainsi qu'aux congés et aux vacances.

6. C'est-à-dire détentrices d'un DEC en techniques de garde ou l'équivalent.

7. Soit les jours fériés, les congés pour obligation familiale, les congés de maladie et les congés personnels.

8. Cette situation n'est d'ailleurs pas propre au Québec. Pour le cas de la France, voir Devetter, 2012. 
comme on l'affirme souvent, certains avantages à exercer cette forme d'emploi atypique susceptible de favoriser la conciliation entre travail et vie familiale? Apprécientelles les marges d'autonomie découlant du fait d'exercer leur activité dans la résidence familiale? Souhaitent-elles le développement d'une relation d'emploi classique, avec la subordination qui la caractérise? Quelles voies privilégient-elles pour le transformer? S’agit-il de préserver le statut de travailleuse autonome tout en améliorant ses conditions d'exercice, d'obtenir des conditions de travail et de rémunération équivalentes à celles des salariées des $\mathrm{CPE}$, ou plutôt d'accroître la reconnaissance (et le niveau de rémunération) par la professionnalisation de l'activité?

La littérature scientifique sur le travail de soins révèle que, loin de constituer un groupe monolithique, les travailleuses du care se distinguent par leur rapport au travail et par l'appréciation subjective qu'elles ont de leur emploi (Cartier et al., 2012, Avril, 2003, 2012). De la même manière que les emplois de soins ne sont pas interchangeables, car associés à des degrés variables de subordination ainsi qu'à des contraintes et des ressources différentes (Devetter, 2012; Avril et Cartier, 2014), les travailleuses exerçant une même activité ne présentent pas les mêmes "propriétés sociales». Les axes de différenciation concernent principalement la scolarité, le statut de l'emploi antérieur, les caractéristiques de la trajectoire professionnelle et les ressources matérielles et culturelles qu'elle a ou non permis de constituer, la présence d'autres sources de revenus dans le ménage et finalement le statut d'immigrante (ou l'appartenance ethnoculturelle), associé à un déclassement professionnel (Devetter, 2012; Cartier et al., 2012; Avril, 2003, 2012).

La croissance des effectifs des travailleurs indépendants est également marquée par une très grande hétérogénéité, qu'il s'agisse de la qualification et du revenu (Marler, Barringer et Milkovich, 2002; Inkson et Arthur, 2001; Peel et Inkson, 2004), du degré réel d'autonomie dont jouissent ces travailleurs (D’Amours et Crespo, 2004), de leur appartenance sexuelle et ethnoculturelle ou des contextes présidant à leur entrée dans cette forme d'emploi (Delage, 2002).

Les travaux scientifiques sur le travail indépendant féminin ont expliqué la récente propension féminine à s'établir comme indépendantes par le désir, présent en particulier chez les mères d'enfants en bas âge, d'une forme d'emploi flexible permettant d'articuler travail rémunéré et vie familiale. Selon Carr (1996), il s'agit d'une voie d'échappement prise par les femmes (notamment celles qui sont scolarisées et qui peuvent compter sur le revenu d'un conjoint) relativement au peu de flexibilité dans le secteur salarié. Le «choix» d'exercer sous ce mode et les motivations pour ce faire sont révélateurs d'arrangements familiaux associant un travailleur dit typique (bénéficiant d'avantages sociaux pour lui-même et sa famille) et une travailleuse atypique, dont l'emploi, par ailleurs faiblement rémunéré et peu protégé, facilite la conciliation entre travail et vie familiale. Mais cette division du travail au sein du couple comporte des effets pervers pour les travailleuses aux prises avec de faibles rémunérations et une dépendance à l'égard des régimes d'assurance de leurs conjoints (Vosko et Zukewich, 2006). 
Les contributions cherchant à expliquer la forte propension des immigrants et des membres des minorités raciales ou culturelles à investir le travail indépendant ont produit des résultats contrastés. Certains (Maxim, 1992; Tang, 1995) sont venus étayer l'hypothèse d'un choix par défaut, selon laquelle les membres de ces groupes seraient «poussés» vers le travail indépendant par la discrimination dont ils font l'objet et les obstacles, notamment linguistiques, qu'ils rencontrent sur le marché du travail salarié. D'autres (Aldrich et Waldinger, 1990) attribuent plutôt ce phénomène à l'existence, dans ces communautés, de forts réseaux familiaux et sociaux générateurs de ressources facilitant l'établissement dans ce type d'arrangement de travail. De nombreux travaux (voir notamment Jurik, 1998, et les contributions réunies par Apitzsch et Kontos, 2003) font ressortir le caractère paradoxal, marqué à la fois par l'exploitation et par l'empowerment, du travail indépendant chez les groupes socialement marginalisés.

Alors que de nombreux travaux ont reposé sur l'analyse d'un rapport de domination principal, voire unique (de classe, de genre ou de race/ethnicité), une documentation foisonnante sur l'intersectionnalité renverse la perspective en proposant que «la différenciation sociale et l'inégalité complexe» (Bilge, 2010), mais aussi les identités et les luttes des groupes dominés pouvaient être mieux comprises à partir d'une analyse en termes d'articulation, d'imbrication ou de coextensivité ${ }^{9}$ des rapports sociaux. L'approche intersectionnelle a été mobilisée notamment dans l'étude du travail de soins (Nakano Glenn, 2008; Cognet, 2010). Sur un registre voisin, la notion de «lieu social» a été proposée par Zavella (1997) pour désigner les espaces sociaux créés par l'intersection de la classe, de l'ethnie, du genre et de l'âge. Elle a été reprise dans les travaux sur les travailleurs précaires (Vosko, 2006) et plus précisément dans l'analyse faite par Jurik (1998) de la manière dont le «lieu social» façonne les possibilités, contraintes et stratégies des travailleurs indépendants du bas de l'échelle.

Nous inspirant de cette dernière perspective, nous avons cherché à comprendre les représentations, les revendications et les aspirations professionnelles, hypothétiquement variées, des RSG, à la lumière de trois axes de structuration des inégalités: la trajectoire professionnelle, les arrangements familiaux et l'expérience de la migration.

\section{MÉTHODOLOGIE ET MODÈLE D'ANALYSE}

Les résultats présentés dans cet article ont été produits dans le cadre d'un projet de recherche réalisé en partenariat ${ }^{10}$ avec la Centrale des syndicats du Québec (CSQ), qui représentait, au moment de l'étude, quelque 13500 des 15000 RSG québécoises.

Les 10348 RSG pour lesquelles nous possédions une adresse électronique ont été sollicitées par courriel pour remplir le sondage administré sur un site sécurisé géré par

9. Pour une analyse des diverses perspectives qui coexistent dans ce courant, voir entre autres les numéros spéciaux de L'Homme et la société (2010/2), en particulier le texte de Bilge, et de Nouvelles pratiques sociales (2014).

10. Les résultats des traitements univariés et bivariés sont consignés dans un rapport de recherche (D’Amours, 2013). Des analyses de régression additionnelles ont été produites pour la rédaction du présent article, qui présente un cadre d'analyse et des résultats originaux. 
le service APTI de l'Université Laval. Les autres ont reçu par la poste une version papier du questionnaire. L'administration du sondage s'est déroulée entre le 26 septembre et le 22 novembre 2011, soit quelques mois à peine après la conclusion d'une première entente collective ${ }^{11}$. Au total, 3769 RSG y ont répondu, dont 770 au moyen du questionnaire papier, pour un taux de réponse de 29,3\%. L'échantillon est conforme à la population des RSG membres de la CSQ eu égard à la variable du sexe, et il a été redressé pour tenir compte de la variable régionale ${ }^{12}$.

Pour le présent article, nous avons construit un modèle d'analyse cherchant à expliquer trois variables dépendantes: le statut préféré (salariat ou indépendance), l'importance accordée à divers types de revendications syndicales (relatives aux conditions de travail, à l'autonomie et à la professionnalisation) et les perspectives de carrière dans le métier (emploi de carrière, emploi transitoire ou emploi par défaut) par trois blocs de variables indépendantes, référant respectivement à la trajectoire professionnelle, à la situation familiale et à l'expérience de la migration. Nous avons formulé trois hypothèses initiales:

Hypothèse 1 : les caractéristiques de la trajectoire professionnelle, à savoir la scolarité, le contexte d'établissement et le nombre d'années d'expérience comme RSG et les caractéristiques de l'emploi antérieur, ont un impact sur le statut préféré, les demandes jugées prioritaires et les perspectives professionnelles des RSG.

Hypothèse 2: les caractéristiques de la situation familiale, à savoir la présence d'un conjoint, le statut de ce conjoint et le soutien économique qu'il peut fournir, le nombre de personnes à charge et la présence d'enfants en bas âge, de même que le revenu familial, ont un impact sur le statut préféré, les demandes jugées prioritaires et les perspectives professionnelles des RSG.

Hypothèse 3 : le processus de migration et la déqualification professionnelle qu'il suppose souvent ont un impact sur le statut préféré, les demandes jugées prioritaires et les perspectives professionnelles des RSG.

Les traitements statistiques que nous exposons dans les sections suivantes ont permis non seulement de vérifier l'existence d'associations statistiquement significatives entre chacun des blocs de variables indépendantes et la majorité des variables dépendantes, mais aussi de constater une forte association entre les variables indépendantes, suggérant l'existence, au sein des RSG, de profils construits à l'intersection de la trajectoire professionnelle, de la situation familiale et de l'expérience de la migration, porteurs de sensibilités et d'aspirations différentes.

11. Nous remercions MM. Jean-Simon Couture, pour la conception et l'administration en ligne du questionnaire, et Gaétan Daigle, statisticien rattaché au département de mathématiques de l'Université Laval, qui a effectué les opérations statistiques nécessaires à la production de ces données. L'auteure demeure toutefois seule responsable de l'analyse des données et des erreurs qui auraient pu s'y glisser.

12. Cette variable est par ailleurs significativement associée à plusieurs autres, notamment le lieu de naissance et la langue maternelle, mais aussi le fait de vivre avec un conjoint et le statut de ce conjoint, de même que le niveau du revenu familial. Les données permettant de contrôler l'échantillon sur d'autres variables, par exemple l'âge, ne sont pas disponibles. 


\section{UN GROUPE HÉTÉROGÈNE QUANT À SES CARACTÉRISTIQUES ET À SES ASPIRATIONS}

Les analyses univariées (fréquences simples) sur chacune des variables indépendantes (voir Tableau 1) permettent de constater une importante hétérogénéité au sein du groupe étudié. En effet, si la quasi-totalité (99,4\%) des RSG sont des femmes, elles se distinguent fortement sur le plan de la scolarité, du secteur mais surtout des caractéristiques de l'emploi antérieur, ainsi que des motifs et de la durée d'établissement comme RSG. Elles sont nombreuses à vivre avec un conjoint mais diffèrent eu égard au statut de ce conjoint et au soutien économique qu'il peut offrir, ainsi qu'aux responsabilités et au revenu familial. Bien que $86,3 \%$ d'entre elles soient nées au Canada, une proportion non négligeable est issue de l'immigration, surtout africaine, latinoaméricaine et asiatique.

La distribution des fréquences des variables dépendantes (voir Tableau 2) indique que les répondantes se partagent à peu près également entre celles qui, si elles avaient le choix, préféreraient être salariées et celles qui préféreraient conserver le statut de travailleuse indépendante. Parmi 10 demandes syndicales, celles relatives aux conditions d'emploi recueillent un appui quasi unanime, alors que celles relatives à l'autonomie et à la professionnalisation recueillent des taux d'adhésion élevés, mais moins unanimes. Finalement, les RSG entretiennent des visions différentes de leur avenir dans cette profession: si certaines le considèrent comme un emploi de carrière, d'autres n'y sont que de passage ou par défaut.

\section{L'IMPACT CONJUGUÉ DU STATUT SOCIOPROFESSION NEL, DE LA SITUATION FAMILIALE ET DE LA MIGRATION}

Comme le révèle le Tableau 3, les traitements bivariés (croisements des variables deux par deux, analysés selon la méthode du chi-deux) révèlent qu'à l'exception notable des demandes concernant les conditions de travail, les autres variables dépendantes sont significativement associées (au seuil de $\mathrm{p}<0,05$ ) avec un grand nombre de variables indépendantes. Des analyses de régression logistique avec fonction de lien logit ont donc été effectuées (voir Tableaux 4 à 11), dans le but d'identifier les variables explicatives détenant le plus fort pouvoir prédictif de certaines variables dépendantes. Pour chacune des variables dépendantes choisies, les variables indépendantes explicatives les plus importantes en régression ont été déterminées par la méthode algorithmique stepwise, qui sélectionne les variables selon leur pouvoir de discrimination entre les deux modalités de la variable dépendante. La statistique c (aire sous la courbe ROC) ${ }^{13}$ informe donc sur l'importance relative des prédicteurs.

13. L'aire sous la courbe ROC est l'équivalent, en régression logistique, du R2 dans un modèle de régression linéaire standard. Comme proposé par Hosmer, Leshow et Sturdivant (2013: 182-186), il s'agit d'une mesure synthèse mieux adaptée que les pseudos R2 pour discriminer les deux états possibles de la variable dépendante. 
Tableau 1 : Tableau de fréquences des variables indépendantes

\begin{tabular}{|c|c|}
\hline \multicolumn{2}{|l|}{ Trajectoire professionnelle } \\
\hline Scolarité (dernier diplôme obtenu') & $\begin{array}{l}\text { Secondaire ou moins: } 49,4 \% \\
\text { Collégial ou universitaire: } 50,6 \%\end{array}$ \\
\hline Contexte de l'établissement comme RSG & $\begin{array}{l}\text { Désir d'être à la maison pour ses enfants tout en gagnant } \\
\text { un revenu }(52,4 \%) \text {; intérêt pour le métier d'éducatrice } \\
(28,5 \%) \text {; désir d'autonomie }(9,2 \%) \text {, horaires de travail } \\
\text { non compatibles avec la vie familiale }(4,9 \%) \text {, emplois } \\
\text { alternatifs moins avantageux que celui de RSG ( } 2,5 \%) \text {, } \\
\text { désir de s'intégrer sur le marché du travail }(2,1 \%) \text {, raisons } \\
\text { fiscales }(0,4 \%) \text {. }\end{array}$ \\
\hline Nombre d'années d'expérience comme RSG & $\begin{array}{l}\text { Moins de } 3 \text { ans : } 8,3 \% \\
\text { De } 3 \text { à } 5 \text { ans: } 16 \% \\
\text { De } 6 \text { à } 10 \text { ans: } 36,4 \% \\
\text { Plus de } 10 \text { ans: } 39,3 \%\end{array}$ \\
\hline $\begin{array}{l}\text { Secteur de l'emploi antérieur occupé le plus } \\
\text { longtemps }\end{array}$ & $\begin{array}{l}\text { Vente et services }(34,6 \%) \text {, administration, incluant les } \\
\text { emplois administratifs et de bureau }(15,7 \%) \text {; sciences } \\
\text { sociales et enseignement }(14,9 \%) \text {; santé }(10,3 \%) ; \\
\text { transformation/fabrication }(9,8 \%) \text {; gestion }(5,9 \%) \text {; } \\
\text { métiers, transport et machinerie }(3,3 \%) \text {; secteur primaire } \\
(2,4 \%) \text {; arts, culture, sports et loisirs }(2,3 \%) \text {; sciences } \\
\text { naturelles et appliquées }(0,8 \%) \text {. }\end{array}$ \\
\hline \multicolumn{2}{|l|}{ Caractéristiques de cet emploi antérieur vs RSC } \\
\hline Rémunération & Plus: $32,3 \%$ Autant: $28,4 \%$ Moins: $39,4 \%$ \\
\hline Protection en cas de chômage & Plus: $74,5 \%$ Autant: $15,2 \%$ Moins: $10,3 \%$ \\
\hline Protection en cas de maladie ou accident & Plus: $59,6 \%$ Autant: $23,0 \%$ Moins : $17,4 \%$ \\
\hline Protection en cas de grossesse & Plus: $62,0 \%$ Autant: $23,0 \%$ Moins: $15,0 \%$ \\
\hline Protection pour la retraite & Plus: $53,7 \%$ Autant: $25,9 \%$ Moins : $20,4 \%$ \\
\hline Possibilité de concilier travail et vie personnelle & Plus: $17,8 \%$ Autant : $28,1 \%$ Moins : $54,1 \%$ \\
\hline Accès à la formation professionnelle & Plus: $20,3 \%$ Autant: $45,0 \%$ Moins : $34,7 \%$ \\
\hline Autonomie au travail & Plus : $6,0 \%$ Autant : $24,5 \%$ Moins : $69,4 \%$ \\
\hline Satisfaction au travail & Plus : $8,5 \%$ Autant : $48,9 \%$ Moins : $42,6 \%$ \\
\hline \multicolumn{2}{|l|}{ Situation familiale } \\
\hline Mariée ou en union libre & $85,5 \%$ \\
\hline Statut d'emploi du conjoint & $\begin{array}{l}\text { Salarié/e syndiqué/e: } 38,1 \% \\
\text { Salarié/e non syndiqué/e : } 39,3 \% \\
\text { Travailleur/euse autonome: } 15,6 \% \\
\text { Sans emploi/Étudiant/e : } 2,3 \% \\
\text { Retraité/e: } 4,7 \%\end{array}$ \\
\hline $\begin{array}{l}\text { Soutien économique du conjoint } \\
\text { en cas de manque de travail ou de maladie }\end{array}$ & $49,2 \%$ des répondantes ayant un conjoint \\
\hline Couverture par les assurances du conjoint & $62,7 \%$ des répondantes ayant un conjoint \\
\hline Nombre de personnes à charge & $\begin{array}{l}\text { Aucune: } 27 \% \\
\text { Une: } 17,3 \% \\
\text { Deux ou plus: } 55,7 \%\end{array}$ \\
\hline
\end{tabular}




\section{Tableau 1: Tableau de fréquences des variables indépendantes (suite)}

\begin{tabular}{|c|c|}
\hline \multicolumn{2}{|l|}{ Situation familiale (suite) } \\
\hline $\begin{array}{l}\text { Nombre d'enfants de moins } \\
\text { de } 6 \text { ans }\end{array}$ & $\begin{array}{l}\text { Aucun: } 74,1 \% \\
\text { Un: } 15,0 \% \\
\text { Deux ou plus: } 10,9 \%\end{array}$ \\
\hline Revenu familial net avant impôt & $\begin{array}{l}\text { Moins de } 20 \text { ooo } \$: 8,4 \% \\
\text { De } 20 \text { à } 39999 \$: 26,3 \% \\
\text { De } 40 \text { à } 59999 \$: 25,1 \% \\
\text { De } 60 \text { à } 79999 \$: 20,8 \% \\
80 \text { ooo } \$ \text { et plus: } 19,4 \%\end{array}$ \\
\hline \multicolumn{2}{|l|}{ Migration } \\
\hline Lieu de naissance & $\begin{array}{l}\text { Canada: } 86,3 \% \text { ( } 84 \% \text { au Québec) } \\
\text { À l'étranger: } 13,7 \% \text { (10,2\% en Afrique, en Amérique latine } \\
\text { ou en Asie) }{ }^{2}\end{array}$ \\
\hline
\end{tabular}

1. Il n'a pas été possible de déterminer si la formation attestée par ce diplôme concerne l'activité de soin et d'éducation des enfants.

2. Dans les traitements statistiques, il n'a pas été possible distinguer les RSC immigrantes selon leur continent d'origine.

\section{Tableau 2: Tableau de fréquences des variables dépendantes}

\begin{tabular}{|c|c|}
\hline Variables dépendantes & Tris à plat \\
\hline Préférence quant au statut & $\begin{array}{l}\text { Préféreraient être salariées : } 43,8 \% \\
\text { Préféreraient conserver le statut de travailleuse } \\
\text { indépendante: } 56,2 \%\end{array}$ \\
\hline \multicolumn{2}{|c|}{ Importance accordée aux demandes relatives aux conditions de travail } \\
\hline Une meilleure rémunération & Très ou assez important: $97,8 \%$ \\
\hline Des congés et des vacances & Très ou assez important: $98,7 \%$ \\
\hline Des assurances collectives et un régime de retraite & Très ou assez important: $93,3 \%$ \\
\hline \multicolumn{2}{|l|}{ Importance accordée aux demandes relatives à l'autonomie } \\
\hline $\begin{array}{l}\text { Le droit de choisir sa clientèle et de déterminer son } \\
\text { programme d'activité }\end{array}$ & Très ou assez important: $93,1 \%$ \\
\hline La limitation des contrôles par l'État & Très ou assez important: $78,4 \%$ \\
\hline \multicolumn{2}{|c|}{ Importance accordée aux demandes relatives à la professionnalisation } \\
\hline Des critères plus exigeants en matière de formation & Très ou assez important: $77,1 \%$ \\
\hline La qualité des services de garde à domicile & Très ou assez important: $97,5 \%$ \\
\hline Perspectives de carrière & $\begin{array}{l}\text { RSG comme emploi de carrière: } 77,5 \% \\
\text { RSG comme choix temporaire: } 14,9 \% \\
\text { RSG comme second choix (préférerait travailler dans un autre } \\
\text { type d'emploi ou ne pas avoir d'emploi rémunéré) : } 7,6 \%\end{array}$ \\
\hline
\end{tabular}




\section{Tableau 3: Traitements bivariés}

\begin{tabular}{|c|c|c|c|c|c|c|c|c|c|}
\hline \multirow[b]{2}{*}{ Famille } & \multirow{2}{*}{$\begin{array}{l}\text { Statut } \\
\text { préféré }\end{array}$} & \multicolumn{3}{|c|}{$\begin{array}{l}\text { Importance accordée aux conditions } \\
\text { d'emploi }\end{array}$} & \multicolumn{2}{|c|}{$\begin{array}{l}\text { Importance accordée } \\
\text { à l'autonomie }\end{array}$} & \multicolumn{2}{|c|}{$\begin{array}{l}\text { Importance accordée } \\
\text { à la professionnalisation }\end{array}$} & \multirow{2}{*}{$\begin{array}{l}\text { Perspectives de carrière } \\
\text { Emploi de carrière, } \\
\text { temporaire ou par défaut }\end{array}$} \\
\hline & & $\begin{array}{l}\text { Rémuné- } \\
\text { ration }\end{array}$ & Congés & $\begin{array}{l}\text { Ass. et rég. } \\
\text { retraite }\end{array}$ & $\begin{array}{l}\text { Choix } \\
\text { clientèle }\end{array}$ & Limite contrôles & $\begin{array}{l}\text { Exigences } \\
\text { formation }\end{array}$ & $\begin{array}{l}\text { Qualité services } \\
\text { de garde }\end{array}$ & \\
\hline Conjoint & - & - & 0,0475 & - & - & 0,0255 & - & - & $<, 0001$ \\
\hline Statut du conjoint & 0,0402 & - & - & - & - & - & - & - & $<, 0001$ \\
\hline Soutien écon. du conjoint & 0,0001 & - & - & - & - & - & - & - & - \\
\hline Assurance conjoint & - & - & - & - & - & - & - & - & 0,0002 \\
\hline Nombre personnes à charge & - & - & - & - & 0,0094 & - & - & - & $<, 0001$ \\
\hline Nombre enfants - de 6 ans & 0,0002 & - & - & - & $<, 0001$ & $<, 0001$ & - & 0,0028 & $<, 0001$ \\
\hline Revenu familial net & 0,0162 & - & - & - & - & - & - & - & 0,0023 \\
\hline \multicolumn{10}{|l|}{ Trajectoire } \\
\hline Scolarité & 0,0479 & - & - & - & 0,0018 & 0,0118 & $<, 0001$ & 0,0419 & $<, 0001$ \\
\hline \multicolumn{10}{|l|}{ Emploi antérieur: } \\
\hline Secteur & - & - & - & - & - & - & 0,0005 & - & $<, 0001$ \\
\hline Rémunération & - & - & - & - & - & - & 0,0174 & - & $<, 0001$ \\
\hline Prot. chômage & - & - & - & - & - & - & - & - & 0,0003 \\
\hline Prot. Mal.-acc. & - & - & - & - & - & - & - & - & 0,0007 \\
\hline Prot. grossesse & - & - & - & - & - & - & - & - & $<, 0001$ \\
\hline Prot. Retraite & - & - & - & - & - & - & - & - & $<, 0001$ \\
\hline Conc T-F & 0,0179 & - & - & - & - & - & - & - & 0,0045 \\
\hline
\end{tabular}




\section{Tableau 3: Traitements bivariés (suite)}

\begin{tabular}{|c|c|c|c|c|c|c|c|c|c|}
\hline \multirow[b]{2}{*}{ Formation prof. } & \multirow{2}{*}{$\begin{array}{r}\begin{array}{l}\text { Statut } \\
\text { préféré }\end{array} \\
-\end{array}$} & \multicolumn{3}{|c|}{$\begin{array}{l}\text { Importance accordée aux conditions } \\
\text { d'emploi }\end{array}$} & \multicolumn{2}{|c|}{$\begin{array}{l}\text { Importance accordée } \\
\text { à l'autonomie }\end{array}$} & \multicolumn{2}{|c|}{$\begin{array}{l}\text { Importance accordée } \\
\text { à la professionnalisation }\end{array}$} & \multirow{2}{*}{$\begin{array}{c}\text { Perspectives de carrière } \\
<, 0001\end{array}$} \\
\hline & & - & - & - & - & - & $<, 0001$ & - & \\
\hline Autonomie & 0,0013 & - & - & 0,0327 & - & - & 0,0005 & - & - \\
\hline Satisfaction & 0,0068 & - & - & - & - & - & - & - & $<, 0001$ \\
\hline Contexte & $<, 0001$ & 0,0058 & - & - & - & 0,019 & - & - & $<, 0001$ \\
\hline Exp. RSG & 0,0001 & - & - & - & $<, 0001$ & $<, 0001$ & - & - & $<, 0001$ \\
\hline \multicolumn{10}{|l|}{ Migration } \\
\hline Lieu de naissance & 0,0002 & - & - & 0,0008 & $<, 0001$ & $<, 0001$ & $<, 0001$ & - & - \\
\hline
\end{tabular}

Lecture: Il y a une association statistique très forte entre le contexte d'établissement comme RSG et le statut d'emploi préféré (<,0001) mais il n'y en a pas entre le secteur de l'emploi antérieur à l'établissement et le statut d'emploi préféré $(-)$. 


\section{Concernant le statut préféré}

La principale variable prédictive d'une préférence pour le statut de travailleuse indépendante est la durée de l'établissement comme RSG (qui explique $54 \%$ du modèle), suivie par le contexte d'établissement (3,5\% du modèle). Les RSG établies depuis plus de 10 ans sont davantage favorables au maintien du statut de travailleuse indépendante, et s'opposent sur ce point aux RSG établies depuis moins de 3 ans. Celles qui se sont établies par désir d'autonomie et, dans une moindre mesure, parce que les emplois qu'elles auraient pu occuper semblaient moins avantageux que celui de RSG, sont plus favorables au maintien du statut d'indépendante que celles qui se sont établies par désir d'être à la maison avec leurs enfants. À l'opposé, celles qui sont devenues RSG pour s'intégrer sur le marché du travail sont significativement moins favorables au statut de travailleuses indépendantes.

\section{Tableau 4: Déterminants de la cote*: de préférence que les RSG continuent d'être considérées comme des travailleuses autonomes}

\begin{tabular}{|c|c|c|c|c|}
\hline Variable & Modalité & $\begin{array}{l}\text { Rapport } \\
\text { de cotes }\end{array}$ & p-valeur & $\begin{array}{c}\text { c } \\
\text { Partiel:*: }\end{array}$ \\
\hline \multirow{4}{*}{$\begin{array}{l}\text { Nombre d'années d'exercice } \\
\text { du métier de RSG }\end{array}$} & Moins de 3 ans (réf) & & & 0,5398 \\
\hline & De 3 à 5 ans & 0,929 & 0,6089 & \\
\hline & De 6 à 10 ans & 1,225 & 0,1173 & \\
\hline & Plus de 10 ans & 1,445 & 0,0044 & \\
\hline \multirow[t]{7}{*}{ Motif principal pour devenir RSG } & $\begin{array}{l}\text { Être à la maison avec } \\
\text { mes enfants (réf.) }\end{array}$ & & & 0,5749 \\
\hline & $\begin{array}{l}\text { Horaires non } \\
\text { compatibles famille }\end{array}$ & 1,029 & 0,8572 & \\
\hline & $\begin{array}{l}\text { Autres emplois moins } \\
\text { avantageux }\end{array}$ & 1,561 & 0,0482 & \\
\hline & Raisons fiscales & 1,421 & 0,5233 & \\
\hline & Intérêt pour le métier & 0,937 & 0,4031 & \\
\hline & $\begin{array}{l}\text { Intégration sur le } \\
\text { marché du travail }\end{array}$ & 0,501 & 0,0046 & \\
\hline & $\begin{array}{l}\text { Plus grande } \\
\text { autonomie }\end{array}$ & 1,852 & $<, 0001$ & \\
\hline
\end{tabular}

* Cette cote est définie comme le rapport de la probabilité de la préférence que les RSG continuent d'être considérées comme des travailleuses autonomes sur le complément de cette probabilité.

** La statistique c (aire sous la courbe ROC) informe sur l'importance relative des prédicteurs.

Lecture: La cote de préférence que les RSG continuent d'être considérées comme des travailleuses autonomes pour les RSG exerçant cette activité depuis plus de 10 ans est de 1,445 fois celle des RSC exerçant l'activité depuis moins de 3 ans. 


\section{Concernant les demandes prioritaires}

Les traitements bivariés témoignent d'un appui massif, et sans beaucoup de distinction selon les variables du modèle, aux revendications relatives à la rémunération, aux congés et vacances, aux assurances collectives et au régime de retraite. À titre exceptionnel, nous avons repéré deux associations significatives, attestant d'une part que les RSG sans conjoint accordent moins d'importance aux congés et aux vacances, et d'autre part que les RSG issues de l'immigration accordent plus d'importance aux assurances collectives et aux régimes de retraite.

Par contre, le degré d'importance attaché aux revendications relatives à l'autonomie et à la professionnalisation varie considérablement selon les caractéristiques de la trajectoire professionnelle, de la situation familiale et du lieu de naissance de la RSG.

Les analyses de régression révèlent en effet que la probabilité de considérer comme très ou assez important le droit de choisir sa clientèle et de déterminer son programme d'activité est prédite d'abord par la présence/l'absence d'enfants de moins de 6 ans (qui explique 57,5\% du modèle) et ensuite par le lieu de naissance de la RSG. Les RSG n'ayant pas d'enfants de moins de 6 ans accordent plus d'importance à cette dimension que celles qui en ont, et les RSG nées au Canada y accordent plus d'importance que celles nées à l'étranger.

\section{Tableau 5: Déterminants de la cote* de considérer comme important ou très important qu'une organisation regroupant les RSG défende le droit de choisir sa clientèle et de déterminer son programme d'activité}

\begin{tabular}{|l|l|c|c|c|}
\hline Variable & Modalité & $\begin{array}{c}\text { Rapport } \\
\text { de cotes }\end{array}$ & p-valeur & $\begin{array}{c}\text { c } \\
\text { Partiel } \%:\end{array}$ \\
\hline $\begin{array}{l}\text { Nombre d'enfants de moins } \\
\text { de } 6 \text { ans }\end{array}$ & $\circ$ (réf.) & & & 0,5752 \\
\cline { 2 - 5 } & 1 & 0,574 & 0,0009 & \\
\cline { 2 - 5 } & 2 ou + & 0,438 & $<, 0001$ & \\
\hline Lieu de naissance & Canada (réf.) & & & 0,6171 \\
\cline { 2 - 5 } & Ailleurs & 0,429 & $<, 0001$ & \\
\hline
\end{tabular}

* Cette cote est définie comme le rapport de la probabilité de considérer comme important ou très important qu'une organisation regroupant les RSG défende le droit de choisir sa clientèle et de déterminer son programme d'activité sur le complément de cette probabilité.

** La statistique c (aire sous la courbe ROC) informe sur l'importance relative des prédicteurs.

Lecture: La cote de considérer comme important ou très important qu'une organisation regroupant les RSC défende le droit de choisir sa clientèle et de déterminer son programme d'activité pour les RSG ayant plus d'un enfant de moins de 6 ans est de 0,438 fois celle des RSG n'en ayant aucun. 
La probabilité de considérer comme très ou assez importante la limitation des contrôles par l'État est prédite d'abord par le lieu de naissance (qui explique 55,6\% du modèle), puis par la durée de l'établissement comme RSG et par la présence/l'absence d'enfants de moins de 6 ans. Les RSG nées au Canada y accordent plus d'importance que celles nées à l'étranger; l'importance croît avec la durée de l'établissement et elle est plus marquée chez les RSG n'ayant pas d'enfants de moins de 6 ans que chez les mères d'un enfant.

\section{Tableau 6: Déterminants de la cote* de considérer comme important ou très important qu'une organisation regroupant les RSG défende la limitation des contrôles par l'État}

\begin{tabular}{|l|l|c|c|c|}
\hline Variable & Modalité & $\begin{array}{c}\text { Rapport } \\
\text { de cotes }\end{array}$ & p-valeur & $\begin{array}{c}\text { c } \\
\text { Partiel } * *\end{array}$ \\
\hline \multirow{2}{*}{ Lieu de naissance } & Canada (réf.) & & & 0,556 \\
\cline { 2 - 5 } & Ailleurs & 0,435 & $<, 0001$ & \\
\hline \multirow{2}{*}{$\begin{array}{l}\text { Nombre d'années d'exercice } \\
\text { du métier de RSC }\end{array}$} & Moins de 3 ans (réf.) & & & 0,614 \\
\cline { 2 - 5 } & De 3 à 5 ans & 1,673 & 0,0011 & \\
\cline { 2 - 5 } & De 6 à 10 ans & 1,499 & 0,0049 & \\
\cline { 2 - 5 } & Plus de 10 ans & 2,209 & $<, 0001$ & \\
\hline \multirow{2}{*}{$\begin{array}{l}\text { Nombre d'enfants de moins } \\
\text { de 6 ans }\end{array}$} & o (réf.) & & & \\
\cline { 2 - 5 } & 1 & 0,698 & 0,0016 & \\
\cline { 2 - 5 } & 2 ou + & 0,1224 & \\
\hline
\end{tabular}

* Cette cote est définie comme le rapport de la probabilité de considérer comme important ou très important qu'une organisation regroupant les RSG défende la limitation des contrôles par l'État sur le complément de cette probabilité. ** La statistique c (aire sous la courbe ROC) informe sur l'importance relative des prédicteurs.

Lecture: La cote de considérer comme important ou très important qu'une organisation regroupant les RSG défende la limitation des contrôles par l'État pour les RSC nées ailleurs qu'au Canada est de o,435 fois celle des RSG nées dans ce pays.

La probabilité de considérer comme très ou assez importants des critères plus exigeants en matière de formation est expliquée en premier lieu par la scolarité (56,8\% du modèle), puis par le lieu de naissance et finalement par le fait que l'emploi antérieur procurait plus d'autonomie que celui de RSG. Les RSG dotées d'une scolarité de niveau collégial ou universitaire, ainsi que celles nées hors du Canada, accordent plus d'importance à cette dimension que celles plus faiblement scolarisées et celles nées au Canada. De même, les RSG à qui l'emploi antérieur procurait plus d'autonomie y accordent plus d'importance et s'opposent à celles dont l'emploi antérieur procurait moins d'autonomie que celui de RSG.

Finalement, la probabilité de considérer comme très ou assez importante la qualité des services de garde à domicile est prédite par une seule variable, la scolarité. De manière à première vue surprenante, ce sont les RSG les moins scolarisées (aucun diplôme ou diplôme secondaire) qui accordent le plus d'importance à cette dimension. 
Tableau 7: Déterminants de la cote* de considérer comme important ou très important qu'une organisation regroupant les RSG défende des critères plus exigeants en matière de formation

\begin{tabular}{|c|c|c|c|c|}
\hline Variable & Modalité & $\begin{array}{l}\text { Rapport } \\
\text { de cotes }\end{array}$ & p-valeur & $\begin{array}{c}\text { C } \\
\text { Partiel } * *\end{array}$ \\
\hline \multirow[t]{2}{*}{ Plus haut diplôme obtenu } & Aucun/Secondaire (réf.) & & & 0,568 \\
\hline & Collégial/Universitaire & 1,634 & $<, 0001$ & \\
\hline \multirow[t]{2}{*}{ Lieu de naissance } & Canada (réf.) & & & 0,582 \\
\hline & Ailleurs & 2,115 & $<, 0001$ & \\
\hline \multirow{3}{*}{$\begin{array}{l}\text { L'emploi exercé avant de devenir } \\
\text { RSG donnait plus, autant, moins de } \\
\text { possibilités de décider du contenu } \\
\text { de mon travail }\end{array}$} & Plus (réf.) & & & 0,591 \\
\hline & Autant & 0,794 & 0,2949 & \\
\hline & Moins & 0,625 & 0,0235 & \\
\hline
\end{tabular}

* Cette cote est définie comme le rapport de la probabilité de considérer comme important ou très important qu'une organisation regroupant les RSG défende des critères plus exigeants en matière de formation sur le complément de cette probabilité.

** La statistique c (aire sous la courbe ROC) informe sur l'importance relative des prédicteurs.

Lecture: La cote de considérer comme important ou très important qu'une organisation regroupant les RSG défende des critères plus exigeants en matière de formation pour les RSG ayant un diplôme collégial ou universitaire est de 1,634 fois celle des RSG n'ayant pas diplôme ou ayant un diplôme du secondaire.

\section{Tableau 8: Déterminants de la cote* de considérer comme important ou très important qu'une organisation regroupant les RSG défende la qualité des services de garde à domicile}

\begin{tabular}{|l|l|c|c|c|}
\hline Variable & Modalité & $\begin{array}{c}\text { Rapport } \\
\text { de cotes }\end{array}$ & p-valeur & $\begin{array}{c}\text { c } \\
\text { Partiel }\end{array}$ \\
\hline Plus haut diplôme détenu & Aucun/Secondaire (réf.) & & & 0,555 \\
\cline { 2 - 5 } & Collégial/Universitaire & 0,647 & 0,0434 & \\
\hline
\end{tabular}

* Cette cote est définie comme le rapport de la probabilité de considérer comme important ou très important qu'une organisation regroupant les RSG défende la qualité des services de garde à domicile sur le complément de cette probabilité.

** La statistique c (aire sous la courbe ROC) informe sur l'importance relative des prédicteurs.

Lecture: La cote de considérer comme important ou très important qu'une organisation regroupant les RSC défende la qualité des services de garde à domicile pour les RSG ayant un diplôme collégial ou universitaire est de o,647 fois celle des RSG n'ayant pas de diplôme ou ayant un diplôme du secondaire.

\section{Concernant la perspective de carrière}

La probabilité de considérer le fait d'être RSG comme un choix de carrière est expliquée par 6 variables qui sont, dans l'ordre: le contexte d'établissement (qui explique $63,8 \%$ du modèle); la présence/l'absence d'enfants de moins de 6 ans; le degré de satisfaction au travail procuré par l'emploi antérieur; la scolarité; le degré de protection en cas de chômage procuré par l'emploi antérieur; et la durée de l'établissement 
Tableau 9: Déterminants de la cote* de considérer le métier de RSG comme un choix de carrière

\begin{tabular}{|c|c|c|c|c|}
\hline Variable & Modalité & $\begin{array}{l}\text { Rapport } \\
\text { de cotes }\end{array}$ & p-valeur & $\begin{array}{c}\text { c } \\
\text { Partiel } * *\end{array}$ \\
\hline \multirow[t]{7}{*}{ Motif principal pour devenir RSG } & Être à la maison avec mes enfants (réf.) & & & 0,638 \\
\hline & Horaires & 1,475 & 0,0439 & \\
\hline & Autres emplois moins avantageux & 1,175 & 0,5396 & \\
\hline & Fiscalité & 1,149 & 0,8344 & \\
\hline & Intérêt pour le métier & 4,215 & $<, 0001$ & \\
\hline & Intégration marché du travail & 0,738 & 0,3389 & \\
\hline & Autonomie & 2,415 & $<, 0001$ & \\
\hline \multirow{3}{*}{$\begin{array}{l}\text { Nombre d'enfants de moins } \\
\text { de } 6 \text { ans }\end{array}$} & o (réf.) & & & 0,694 \\
\hline & 1 & 0,609 & 0,0001 & \\
\hline & $2 \mathrm{ou}+$ & 0,397 & $<, 0001$ & \\
\hline \multirow{3}{*}{$\begin{array}{l}\text { L'emploi précédent me procurait } \\
\text { plus, autant, moins } \\
\text { de satisfaction au travail }\end{array}$} & Plus (réf.) & & & 0,733 \\
\hline & Autant & 2,781 & $<, 0001$ & \\
\hline & Moins & 4,947 & $<, 0001$ & \\
\hline \multirow[t]{2}{*}{ Plus haut diplôme détenu } & Aucun/Secondaire (réf.) & & & 0,746 \\
\hline & Collégial/Universitaire & 0,558 & $<, 0001$ & \\
\hline \multirow{3}{*}{$\begin{array}{l}\text { L'emploi précédent me } \\
\text { procurait plus, autant, moins de } \\
\text { protection en cas de chômage }\end{array}$} & Plus (réf.) & & & 0,750 \\
\hline & Autant & 1,341 & 0,0314 & \\
\hline & Moins & 1,576 & 0,0100 & \\
\hline \multirow{4}{*}{$\begin{array}{l}\text { Nombre d'années d'exercice du } \\
\text { métier de RSC }\end{array}$} & Moins de 3 ans (réf.) & & & 0,755 \\
\hline & De 3 à 5 ans & 0,836 & 0,3186 & \\
\hline & De 6 à 10 ans & 1,013 & 0,9414 & \\
\hline & Plus de 10 ans & 1,376 & 0,0793 & \\
\hline
\end{tabular}

* Cette cote est définie comme le rapport de la probabilité de considérer le métier de RSG comme un choix de carrière sur le complément de cette probabilité.

** La statistique c (aire sous la courbe ROC) informe sur l'importance relative des prédicteurs.

Lecture: La cote de considérer le métier de RSG comme un choix de carrière pour les RSG à qui l'emploi précédent procurait moins de satisfaction est 4,947 fois celle des RSG à qui l'emploi précédent procurait plus de satisfaction.

comme RSG. Cette probabilité est plus grande chez celles qui se sont établies par intérêt pour le métier, par désir d'autonomie et parce que les horaires de travail offerts étaient peu compatibles avec la vie familiale que chez celles qui se sont établies par désir d'être à la maison avec leurs enfants. Elle est plus élevée chez celles qui n'ont pas d'enfants en bas âge que chez celles qui en ont, chez celles à qui l'emploi antérieur procurait autant ou moins de satisfaction, les faiblement scolarisées, celles à qui l'emploi antérieur procurait autant ou moins de protection en cas de chômage et celles établies depuis plus de 10 ans. 
La probabilité de considérer le fait d'être RSG comme un choix temporaire est expliquée quant à elle par 8 variables, dans l'ordre: la présence/l'absence d'enfants de moins de 6 ans (qui explique $66 \%$ du modèle); le contexte d'établissement; le degré de satisfaction procuré par l'emploi antérieur; la scolarité; la durée de l'établissement comme RSG; la couverture par les assurances du conjoint; le degré de protection en cas de chômage procuré par l'emploi antérieur; et le secteur de l'emploi antérieur. Cette probabilité est plus importante chez les RSG ayant au moins deux enfants, et moindrement un enfant de moins de 6 ans, chez celles qui se sont établies par désir d'être à la maison avec leurs enfants (par opposition à celles qui se sont établies par intérêt pour le métier ou par désir d'autonomie), chez celles à qui l'emploi antérieur procurait plus de satisfaction au travail, chez les détentrices d'un diplôme collégial ou universitaire, chez les RSG établies plus récemment, chez celles qui bénéficient d'une couverture par les assurances du conjoint, chez celles dont l'emploi antérieur donnait accès à une meilleure protection en cas de chômage et finalement celles dont l'emploi antérieur était en sciences sociales et enseignement (par opposition à ventes et services et à métiers, transport et machinerie).

Finalement, la probabilité de considérer le fait d'être RSG comme un choix par défaut est expliquée par deux variables: le degré de satisfaction au travail procuré par l'emploi antérieur (expliquant 59,5\% du modèle) et le contexte d'établissement. Cette probabilité est plus grande chez les RSG à qui l'emploi antérieur procurait plus de satisfaction au travail et chez celles qui se sont établies pour des raisons fiscales, pour s'intégrer sur le marché du travail ou parce que les emplois qu'elles auraient pu occuper leur semblaient moins avantageux que celui de RSG, comparées aux RSG qui se sont établies pour être à la maison avec leurs enfants.

\section{DISCUSSION}

Si les responsables de services de garde en milieu familial peuvent être vues comme des travailleuses atypiques vulnérables, surtout si on compare leur situation à celle des éducatrices salariées des CPE, force est de constater qu'elles se distinguent grandement entre elles quant à l'appréciation qu'elles ont de leur situation, aux moyens qu'elles privilégient pour l'améliorer et à leurs perspectives professionnelles.

Des résultats précédemment exposés, il est possible de tirer les conclusions suivantes. D'une part, des variables opérationnalisant chacune des trois hypothèses explicatives ont un impact sur les variables dépendantes. D'autre part, et plus fondamentalement, il est impossible d'associer les différences à un seul bloc de variables indépendantes. Au contraire, les analyses de régression témoignent du rôle conjugué joué par des variables associées à la trajectoire, à la situation familiale et à la migration pour prédire le statut préféré, l'importance accordée à certaines revendications et les perspectives professionnelles des RSG. Ce dernier constat de même que celui d'une forte association statistique entre les variables indépendantes des différents blocs nous amènent, dans cette dernière section, à expliquer l'hétérogénéité des demandes et 
Tableau 10: Déterminants de la cote** de considérer le métier de RSG comme un choix temporaire

\begin{tabular}{|c|c|c|c|c|}
\hline Variable & Modalité & $\begin{array}{l}\text { Rapport } \\
\text { de cotes }\end{array}$ & p-valeur & $\begin{array}{c}\text { C } \\
\text { Partiel } * *\end{array}$ \\
\hline \multirow{3}{*}{$\begin{array}{l}\text { Nombre d'enfants de moins } \\
\text { de } 6 \text { ans }\end{array}$} & o (réf.) & & & 0,662 \\
\hline & 1 & 2,070 & $<, 0001$ & \\
\hline & $2 \mathrm{ou}+$ & 3,226 & $<, 0001$ & \\
\hline \multirow{7}{*}{$\begin{array}{l}\text { Raison principale qui vous a } \\
\text { amenée à devenir RSG }\end{array}$} & Être à la maison avec mes enfants (réf.) & & & 0,742 \\
\hline & Horaires & 0,757 & 0,2227 & \\
\hline & Autres emplois moins avantageux & 0,603 & 0,1554 & \\
\hline & Fiscalité & 0,457 & 0,4667 & \\
\hline & Intérêt pour le métier & 0,182 & $<, 0001$ & \\
\hline & Intégration marché du travail & 0,933 & 0,8706 & \\
\hline & Autonomie & 0,111 & $<, 0001$ & \\
\hline \multirow{3}{*}{$\begin{array}{l}\text { L'emploi précédent me procurait } \\
\text { plus, autant, moins } \\
\text { de satisfaction au travail }\end{array}$} & Plus (réf.) & & & 0,766 \\
\hline & Autant & 0,627 & 0,0102 & \\
\hline & Moins & 0,368 & $<, 0001$ & \\
\hline \multirow[t]{2}{*}{ Plus haut diplôme détenu } & Aucun/Secondaire (réf.) & & & 0,778 \\
\hline & Collégial/Universitaire & 1,736 & $<, 0001$ & \\
\hline \multirow{4}{*}{$\begin{array}{l}\text { Nombre d'années d'exercice du } \\
\text { métier de RSG }\end{array}$} & Moins de 3 ans (réf.) & & & 0,790 \\
\hline & De 3 à 5 ans & 1,129 & 0,5545 & \\
\hline & De 6 à 10 ans & 0,826 & 0,3401 & \\
\hline & Plus de 10 ans & 0,469 & 0,0009 & \\
\hline \multirow{2}{*}{$\begin{array}{l}\text { Êtes-vous couverte par les } \\
\text { assurances de votre conjoint/e? }\end{array}$} & Oui (réf.) & & & 0,794 \\
\hline & Non & 0,693 & 0,0046 & \\
\hline \multirow{3}{*}{$\begin{array}{l}\text { Mon emploi précédent } \\
\text { m'offrait plus, autant, moins de } \\
\text { protection en cas de chômage }\end{array}$} & Plus (réf.) & & & 0,797 \\
\hline & Autant & 0,695 & 0,0409 & \\
\hline & Moins & 0,531 & 0,0138 & \\
\hline \multirow[t]{6}{*}{ Secteur d'emploi antérieur } & Sc. sociales, enseignement (réf.) & & & 0,803 \\
\hline & Gestion & 0,667 & 0,1443 & \\
\hline & Finance et administration & 0,715 & 0,1049 & \\
\hline & Sc. Nat. et appliquées & 0,864 & 0,7825 & \\
\hline & Sc. santé & 0,975 & 0,9083 & \\
\hline & Arts-culture & 0,524 & 0,1066 & \\
\hline
\end{tabular}


Tableau 10: Déterminants de la cote* de considérer le métier de RSG comme un choix temporaire (suite)

\begin{tabular}{|l|l|c|c|c|}
\hline Variable & Modalité & $\begin{array}{c}\text { Rapport } \\
\text { de cotes }\end{array}$ & p-valeur & $\begin{array}{c}\text { c } \\
\text { Partiel } * *\end{array}$ \\
\hline \multirow{2}{*}{$\begin{array}{l}\text { Secteur d'emploi antérieur } \\
\text { (suite })\end{array}$} & Ventes-services & 0,666 & 0,028 & \\
\cline { 2 - 5 } & Métiers-transport & 0,160 & 0,0007 & \\
\cline { 2 - 6 } & Secteur primaire & 0,471 & 0,0844 & \\
\cline { 2 - 6 } & Transformation/fabrication & 0,820 & 0,4252 & \\
\hline
\end{tabular}

* Cette cote est définie comme le rapport de la probabilité de considérer le métier de RSG comme un choix temporaire sur le complément de cette probabilité.

** La statistique c (aire sous la courbe ROC) informe sur l'importance relative des prédicteurs.

Lecture: La cote de considérer le métier de RSG comme un choix temporaire pour les RSG à qui l'emploi précédent procurait moins de satisfaction est 0,368 fois celle des RSC à qui l'emploi précédent procurait plus de satisfaction.

\section{Tableau 11: Déterminants de la cote* de considérer le métier de RSG comme un second choix}

\begin{tabular}{|l|l|l|l|l|}
\hline Variable & Modalité & $\begin{array}{c}\text { Rapport } \\
\text { de cotes }\end{array}$ & p-valeur & $\begin{array}{c}c \\
\text { Partiel } *:\end{array}$ \\
\hline \multirow{2}{*}{$\begin{array}{l}\text { L'emploi précédent me } \\
\text { procurait plus, autant, moins de } \\
\text { satisfaction au travail }\end{array}$} & Plus (réf.) & & & 0,595 \\
\cline { 2 - 6 } & Autant & 0,391 & $<, 0001$ & \\
\cline { 2 - 6 } & Moins & 0,267 & $<, 0001$ & \\
\hline \multirow{2}{*}{$\begin{array}{l}\text { Raison principale qui vous a } \\
\text { amenée à devenir RSG }\end{array}$} & Être à la maison avec mes enfants (réf.) & & & \multirow{2}{*}{0,652} \\
\cline { 2 - 6 } & Horaires & 0,438 & 0,0534 & \\
\cline { 2 - 6 } & Autres emplois moins avantageux & 2,009 & 0,0443 & \\
\cline { 2 - 6 } & Fiscalité & 3,403 & 0,1298 & \\
\cline { 2 - 6 } & Intérêt pour le métier & 0,441 & 0,0004 & \\
\hline & Intégration m du t & 2,487 & 0,0234 & \\
\hline & Autonomie & 1,123 & 0,6507 & \\
\hline
\end{tabular}

* Cette cote est définie comme le rapport de la probabilité de considérer le métier de RSC comme un second choix sur le complément de cette probabilité.

** La statistique c (aire sous la courbe ROC) informe sur l'importance relative des prédicteurs.

Lecture: La cote de considérer le métier de RSG comme un second choix pour les RSG à qui l'emploi précédent procurait moins de satisfaction est 0,267 fois celle des RSC à qui l'emploi précédent procurait plus de satisfaction. 
aspirations des RSG par l'imbrication de leur trajectoire professionnelle, de leur situation familiale et de leur expérience de la migration.

\subsection{Des lieux sociaux construits à l'intersection de la trajectoire, de la famille et de la migration}

Nous avons vu (section 4) que les RSG présentent des différences majeures, d'une part eu égard à leur scolarité et à leur trajectoire antérieure sur le marché du travail, et d'autre part à leur situation familiale. Les croisements des variables indépendantes entre elles (voir tableau 12) révèlent que ces deux blocs de variables sont imbriqués, traçant les contours de deux grands profils parmi les répondantes.

D'un côté, on retrouve les RSG plus âgées, n'ayant pas ou plus d'enfants en bas âge, moins scolarisées mais disposant d'une plus longue expérience professionnelle à titre de RSG et plus nombreuses à avoir exercé auparavant des emplois administratifs et de bureau ou encore à avoir travaillé en usine. Les plus âgées semblent aussi davantage fragilisées par leur situation familiale, étant plus nombreuses à vivre sans conjoint ou avec un conjoint retraité ou travailleur autonome. De l'autre, on retrouve les RSG d'établissement récent, conjointes d'un travailleur salarié et mères de jeunes enfants, qui s'avèrent proportionnellement plus nombreuses à détenir un diplôme postsecondaire et à avoir travaillé dans le secteur des sciences sociales et de l'enseignement (dans lequel nous incluons les services de garde en CPE). De manière générale, les RSG sans personne à charge ont occupé antérieurement des emplois présentant des conditions plus défavorables que celles de RSG ayant au moins deux personnes à charge.

L'imbrication de la trajectoire professionnelle et de la division sociale du travail au sein de la famille apparaît de manière éclatante dans les motifs que les répondantes citent pour expliquer leur établissement comme RSG. Il existe d'une part de nombreuses et fortes associations entre les caractéristiques familiales et le motif d'établissement. Notamment, le motif de s'établir pour être avec les enfants est moins présent chez les RSG dont le conjoint est retraité et, dans une moindre mesure, autonome, sans emploi ou étudiant, et plus présent chez celles dont le conjoint est un travailleur non syndiqué (égal chez les conjointes de syndiqués); il est aussi plus présent chez celles qui peuvent compter sur le soutien économique du conjoint, sont couvertes par ses assurances et disposent d'un revenu familial de 80000 \$ ou plus; il augmente avec le nombre de personnes à charge et la présence d'enfants en bas âge. L'établissement par désir d'autonomie est plus souvent cité par les répondantes sans conjoint ou vivant avec un conjoint précaire, ne pouvant compter sur le soutien économique de celui-ci, sans personne à charge ni enfants en bas âge, et ce motif d'établissement est sousreprésenté parmi les tranches de revenu familial élevées. Il existe d'autre part une association forte entre le motif d'établissement, le secteur de l'emploi antérieur et certaines caractéristiques de cet emploi. Le motif «être à la maison avec les enfants» est surreprésenté dans les emplois administratifs et de bureau; le motif des horaires de travail peu compatibles avec la vie familiale est surreprésenté en milieu de la santé; les motifs «intérêt pour le métier» et «désir d'autonomie» sont quant à eux surreprésen- 
Tableau 12: Croisements des variables indépendantes entre elles (chi-deux)

\begin{tabular}{|c|c|c|c|c|c|c|c|c|}
\hline & \multicolumn{7}{|c|}{ Famille } & \multirow{2}{*}{$\begin{array}{c}\text { Migration } \\
\text { Lieu de } \\
\text { naissance }\end{array}$} \\
\hline VI & Conjoint & $\begin{array}{l}\text { Statut du } \\
\text { conjoint }\end{array}$ & $\begin{array}{l}\text { Soutien } \\
\text { écon. } \\
\text { conjoint }\end{array}$ & $\begin{array}{c}\text { Ass. } \\
\text { conjoint }\end{array}$ & $\begin{array}{l}\text { Nombre } \\
\text { pers. à } \\
\text { charge }\end{array}$ & $\begin{array}{l}\text { Nombre } \\
\text { enfants } \\
-6 \text { ans }\end{array}$ & $\begin{array}{c}\text { Revenu } \\
\text { familial } \\
\text { net }\end{array}$ & \\
\hline \multicolumn{9}{|l|}{ Famille } \\
\hline Conjoint & & & & & & & & - \\
\hline Statut du conjoint & & & & & & & & $<, 0001$ \\
\hline $\begin{array}{l}\text { Soutien écon. } \\
\text { conjoint }\end{array}$ & & & & & & & & $<, 0001$ \\
\hline Ass. conjoint & & & & & & & & $<, 0001$ \\
\hline $\begin{array}{l}\text { Nombre pers. } \\
\text { à charge }\end{array}$ & & & & & & & & $<, 0001$ \\
\hline $\begin{array}{l}\text { Nombre enfants - } \\
6 \text { ans }\end{array}$ & & & & & & & & 0,0043 \\
\hline Revenu familial net & & & & & & & & $<, 0001$ \\
\hline \multicolumn{9}{|l|}{ Trajectoire } \\
\hline Scolarité & - & 0,0039 & 0,0108 & - & $<, 0001$ & $<, 0001$ & 0,0122 & $<, 0001$ \\
\hline \multicolumn{9}{|l|}{ Emploi antérieur: } \\
\hline Secteur & - & 0,0124 & - & - & - & 0,0005 & 0,0004 & $<, 0001$ \\
\hline Rémunération & - & - & - & - & 0,0014 & 0,0005 & 0,0359 & 0,0147 \\
\hline Prot. chômage & - & - & 0,0025 & 0,0249 & $<, 0001$ & - & - & 0,0006 \\
\hline Prot. Mal.-acc. & - & - & - & 0,0003 & $<, 0001$ & 0,0010 & - & 0,0015 \\
\hline Prot. grossesse & 0,0027 & 0,0003 & - & 0,0004 & $<, 0001$ & $<, 0001$ & $<, 0001$ & - \\
\hline Prot. retraite & - & - & 0,0451 & - & $<, 0001$ & $<, 0001$ & - & $<, 0001$ \\
\hline Conc T-F & 0,0003 & 0,0023 & - & - & $<, 0001$ & $<, 0001$ & $<, 0001$ & $<, 0001$ \\
\hline $\begin{array}{l}\text { Formation } \\
\text { Prof. }\end{array}$ & - & 0,0156 & 0,0329 & - & 0,0005 & - & - & $<, 0001$ \\
\hline Autonomie & - & - & - & - & 0,0015 & - & 0,0001 & $<, 0001$ \\
\hline Satisfaction & - & - & - & - & 0,0005 & - & 0,0269 & $<, 0001$ \\
\hline Contexte & $<, 0001$ & $<, 0001$ & 0,0002 & $<, 0001$ & $<, 0001$ & $<, 0001$ & $<, 0001$ & $<, 0001$ \\
\hline Exp. RSG & $<, 0001$ & $<, 0001$ & 0,0003 & - & $<, 0001$ & $<, 0001$ & 0,0001 & $<, 0001$ \\
\hline
\end{tabular}

tés en sciences sociales et enseignement. De manière générale, les RSG qui déclarent s'être établies par désir d'autonomie et par intérêt pour le métier associent leur emploi antérieur à des caractéristiques plus défavorables que celles qui se sont établies par désir d'être à la maison avec leurs enfants, sauf pour la conciliation entre travail et vie personnelle. 
Le contexte d'établissement, les caractéristiques familiales, la scolarité et certains attributs de la trajectoire antérieure se conjuguent finalement pour dessiner des perspectives de carrière contrastées. Comme le révèlent les analyses de régression, le fait de considérer le statut de RSG comme un choix temporaire se retrouve chez les mères de jeunes enfants s'étant établies pour être à la maison avec eux et bénéficiant de la couverture par les assurances du conjoint. Ces travailleuses plus jeunes et plus scolarisées, hypothétiquement plus mobiles sur le marché du travail, considèrent que leur emploi antérieur était plus satisfaisant, et à certains égards plus protecteur, que celui de RSG et envisagent donc, une fois que leurs enfants auront atteint l'âge de la fréquentation scolaire, de retrouver un emploi comparable. À l'inverse, le fait de considérer le statut de RSG comme un choix de carrière est plus répandu chez les répondantes établies par intérêt pour le métier ou par désir d'autonomie, plus âgées que les précédentes, sans enfants en bas âge, et cumulant les caractéristiques professionnelles défavorables: faible scolarité, longue expérience comme RSG, donc caractère daté des expériences professionnelles autres et à qui l'emploi antérieur procurait des conditions moins favorables que celles procurées par leur statut actuel. En outre, ces répondantes estiment que l'activité de RSG leur apporte plus de satisfaction et, à certains égards, plus de protection, que leur emploi antérieur.

Mais cette imbrication de la trajectoire professionnelle et de la situation familiale apparaît sous un angle bien différent lorsqu'on cible les RSG issues de l'immigration, que leurs caractéristiques distinguent des RSG «de souche» et qui, de ce fait, sont porteuses de représentations et d'aspirations particulières.

Les RSG nées à l'étranger s'établissent proportionnellement moins pour être à la maison avec leurs enfants et davantage par désir d'intégration, d'autonomie et, dans une moindre mesure, par intérêt pour le métier d'éducatrice. Elles sont beaucoup plus nombreuses que les RSG nées au Canada à détenir un diplôme universitaire et à avoir travaillé dans le secteur des services sociaux et de l'enseignement. L'emploi antérieur à leur établissement comme RSG présentait de manière générale des caractéristiques plus favorables que leur emploi actuel: il n'était pas moins bien rémunéré et il offrait davantage de protection pour la retraite, de possibilité de concilier travail et vie familiale, de formation, d'autonomie et de satisfaction. Bref, la transition vers le statut de RSG a signifié pour elles une détérioration de leurs conditions de travail, ce qui n'est pas le cas pour une partie des RSG nées au Canada, notamment les plus expérimentées, qui sont aussi les plus âgées et les moins scolarisées. Par ailleurs, les RSG nées à l'étranger sont aussi plus vulnérables en raison du faible niveau de ressources fournies par la famille: revenu familial plus bas, statut plus précaire du conjoint et moindre possibilité de compter sur son revenu, moindre couverture par ses assurances et un plus grand nombre de personnes à charge. La déqualification professionnelle consécutive à la migration amène ces RSG pourtant scolarisées à considérer leur état, non pas comme transitoire (ce qui est le cas des RSG scolarisées nées au Canada) mais plutôt comme un choix par défaut. En effet, le fait de considérer le métier de RSG comme un second choix est prédit par l'évaluation que l'emploi antérieur procurait plus de satisfaction 
que l'emploi de RSG et par un établissement pour des motifs d'intégration sur le marché du travail et d'emplois alternatifs moins favorables, ce qui correspond en partie aux caractéristiques des RSG issues de l'immigration.

\subsection{Lieu social et revendications particulières}

L'histoire récente des associations de RSG au Québec met en lumière plusieurs avenues possibles pour l'amélioration de leurs conditions de travail (D'Amours, 2010; Bisson 2012). Alors que certaines revendiquent la parité avec les éducatrices des CPE, d'autres souhaitent le retour à l'autonomie dont elles jouissaient avant la réforme de 1997, couplé à une bonification des conditions d'exercice, notamment de la rémunération; d'autres encore identifient dans la professionnalisation la voie rêvée pour améliorer tant les conditions de travail que le degré d'autonomie dans l'exercice de l'activité.

L'adhésion marquée (supérieure à 90\%) à plusieurs demandes formulées par la CSQ lors des négociations de 2011 peut s'expliquer par le fait que les RSG «partaient de loin» et qu'elles ont fait front commun derrière les revendications syndicales. Toutefois, contrairement aux demandes portant sur les conditions de travail, celles relatives à l'autonomie et à la professionnalisation fluctuent selon les variables du modèle, et il en va de même pour le statut d'emploi préféré. Il apparaît ainsi que l'appréciation que les RSG ont de leur travail et de leur emploi ainsi que les voies qu'elles privilégient pour l'améliorer ne découlent pas mécaniquement des conditions objectives de travail prévalant dans la garde d'enfants à domicile. Nous nous intéresserons particulièrement ici aux caractéristiques des RSG qui cherchent à maintenir les marges d'autonomie procurées par leur statut et de celles qui priorisent les demandes que nous avons associées à la professionnalisation.

Rappelons que les RSG sont divisées quant au statut d'emploi préféré: si elles avaient le choix, 43,8\% préféreraient être considérées comme des salariées, un statut globalement plus avantageux du point de vue des conditions de travail, alors que $56,2 \%$ souhaiteraient continuer d'être considérées comme des travailleuses autonomes. Comme nous l'avons vu précédemment (Tableau 4), la préférence pour le statut de travailleuse autonome est plus marquée chez les RSG les plus expérimentées et chez celles établies par désir d'autonomie, parce que les emplois alternatifs étaient moins avantageux ou pour des raisons fiscales. Une première piste d'explication possible est bien sûr que les RSG les plus expérimentées ont plus de chances d'avoir connu l'époque antérieure à la réforme de 1997, où leur marge de manœuvre était beaucoup plus grande qu'elle ne l'est devenue par la suite. Mais une deuxième piste d'explication surgit lorsqu'on examine les déterminants de l'importance accordée aux demandes relatives à l'autonomie, dont on a pu constater qu'elle est le fait des RSG sans enfants en bas âge (en général plus âgées et plus expérimentées) et des Québécoises de souche, un profil associé à des conditions moins favorables, en termes de scolarité, que celui des mères de jeunes enfants et des RSG issues de l'immigration. Ce constat voulant que les RSG les plus vulnérables soient aussi celles qui accordent le plus d'importance à 
l'autonomie ne devrait pourtant pas surprendre. L'étude d'Avril et Cartier (2014) fait ainsi ressortir que les travailleuses peu qualifiées apprécient les marges de liberté et d'autonomie offertes dans certains emplois à domicile (autonomie quant au rythme et à la manière d'effectuer le travail, quasi-absence de surveillance directe), parce qu'elles contrastent avec le fort degré de prescription et la surveillance étroite qui caractérisent les emplois féminins faiblement qualifiés, en usines ou dans les services. Bref, on peut faire l'hypothèse que, sur une base comparative avec les emplois que les RSG plus âgées et faiblement scolarisées ont occupés antérieurement, ou des emplois qu'elles pourraient raisonnablement occuper, le statut de RSG offre souvent plus d'autonomie et de satisfaction.

Les demandes relatives à la professionnalisation lèvent quant à elles le voile sur un intéressant paradoxe. Les RSG scolarisées (et les immigrantes, qui sont en moyenne plus scolarisées que les Québécoises de souche) se révèlent plus favorables à l'accroissement des critères de scolarité requis pour exercer l'activité, ce qui se conçoit fort bien puisque l'exigence de posséder des qualifications reconnues par un diplôme pourrait servir d'argument de négociation pour bonifier leur rémunération. C’est aussi le cas, quoique dans une moindre mesure, pour celles à qui l'emploi antérieur procurait davantage d'autonomie. Par ailleurs, l'importance accordée à la qualité des services de garde à domicile est prédite par la seule variable de la scolarité mais, contrairement à ce qu'on aurait pu anticiper, cette affirmation est le fait des RSG les moins scolarisées. Ce résultat à première vue étonnant peut être interprété comme une affirmation et une demande de reconnaissance de la qualité du service que ces RSG rendent, en dépit de leur faible scolarité. Pour elles, la qualité du service qu'elles offrent est ancrée, non pas dans une scolarité qu'elles ne possèdent pas, mais dans leur expérience de mère et leurs longues années d'expérience dans l'exercice de la garde d'enfants à domicile (Bisson, 2012).

Finalement, l'expérience de la migration et la déqualification professionnelle qu'elle suppose souvent ont aussi un effet sur l'évaluation que les travailleuses de la garde à domicile ont de leur emploi et de leur statut. Tout comme les RSG d'établissement récent, les répondantes issues de l'immigration et celles qui sont devenues RSG pour s'intégrer au marché du travail affichent une préférence pour le statut de salariée. Elles sont plus nombreuses à trouver important l'accès à des assurances collectives et à des régimes de retraite et à vouloir hausser les critères de formation, hypothétiquement associés à une meilleure rémunération. Par contre, elles sont plus nombreuses à ne pas trouver très importants les attributs de l'autonomie, ce qui est cohérent avec la priorité accordée à l'amélioration des conditions de travail, de la part de travailleuses très scolarisées mais fragilisées par leur situation personnelle et familiale, que reflète notamment la faiblesse de leur revenu familial annuel.

\section{CONCLUSION}

Le rapport social de travail (D’Amours, 2015) dans le secteur de la garde d'enfants à domicile apparaît comme un hybride entre salariat et indépendance, une forme de 
travail atypique, faiblement rémunéré et peu protégé par les lois du travail, impliquant d'importantes disparités de traitement avec les éducatrices salariées des CPE. Ce constat ne permet pourtant pas de prédire l'appréciation que les travailleuses concernées en ont ni leurs aspirations et revendications professionnelles. Notre étude révèle que les représentations que les RSG se font de leur situation, l'importance qu'elles attribuent à certaines revendications et la manière dont elles se projettent dans l'avenir sont variables. Elles peuvent être expliquées par l'imbrication de trois axes de structuration des inégalités: la trajectoire professionnelle; la division sexuelle du travail au sein de la famille mais également les ressources fournies par la famille; et l'expérience de la migration.

En effet, non seulement les variables choisies pour opérationnaliser ces axes ressortent-elles avec force pour expliquer le statut préféré, les demandes prioritaires et les perspectives professionnelles mais plusieurs sont statistiquement associées entre elles. Pour le dire succinctement, les RSG les plus expérimentées, qui cumulent des caractéristiques défavorables sur le plan de la trajectoire et une situation familiale souvent vulnérable, manifestent un fort attachement à l'autonomie. Relativement satisfaites de leur situation, en l'absence d'alternative plus favorable, elles envisagent de faire carrière comme RSG. Les mères de jeunes enfants, plus jeunes et plus scolarisées, ont quitté des emplois aux caractéristiques plus favorables que celui de RSG (sauf en matière de conciliation travail-famille) pour s'occuper temporairement de leur progéniture et prévoient quitter ce statut une fois que leurs enfants auront atteint l'âge de la fréquentation scolaire. Elles accordent de l'importance aux conditions de travail (notamment aux congés et aux vacances) et souhaitent l'imposition de critères de scolarité plus exigeants, qui leur permettraient de négocier une rémunération plus élevée. Les RSG issues de l'immigration partagent avec les précédentes les conditions plus favorables de l'emploi antérieur, la forte scolarité et les demandes jugées prioritaires mais s'en distinguent par une situation familiale fragile et par la vision d'un avenir bloqué, qui en fait des RSG par défaut.

Une partie des données que nous avons exposées peut être analysée par le "choix», tributaire de la division sexuelle du travail au sein de la famille, d'un certain nombre de jeunes mères scolarisées de s'établir temporairement comme RSG pour être à la maison avec leurs enfants, tendant à accréditer les résultats produits par les travaux de Carr (1996). L'établissement d'une autre partie des RSG n'a rien à voir avec la conciliation entre travail et vie familiale mais découle d'une trajectoire antérieure défavorable et du peu d'options alternatives. Ces RSG plus âgées et moins scolarisées ont une expérience plus lointaine du marché du travail, dans des emplois dont les caractéristiques sont moins favorables que celles de leur statut actuel. Leur situation s'inscrit dans la tradition des travaux qui ont analysé la croissance du travail indépendant comme la manifestation de la précarisation des emplois occupés par des travailleuses faiblement qualifiées, tout en mettant en évidence l'aspiration de ces travailleuses à bénéficier de marges d'autonomie qui leur sont le plus souvent déniées dans les emplois qu'elles ont précédemment occupés. Le profil des RSG issues de l'immigration 
diffère des deux précédents. Elles sont scolarisées et leurs emplois antérieurs leur procuraient des conditions plus favorables que leur statut actuel, ce qui suggère une déqualification professionnelle consécutive à la migration, mais ne voient pas leur état comme transitoire.

Cet article a pu contribuer à éclairer la réalité des RSG par le regard «intérieur» offert par la subjectivité des travailleuses, complémentaire à celui qu’il est possible d'obtenir par l'analyse «extérieure» du rapport social de travail, le premier ne découlant pas mécaniquement du second. Un autre apport consiste à avoir mis en évidence le fait que l'aspiration à l'autonomie dans le travail n'est pas l'apanage des travailleurs fortement qualifiés mais également de travailleuses faiblement qualifiées. Il permet d'appréhender l'hétérogénéité des situations et des représentations du métier qui coexistent au sein du groupe des RSG, qui annonce déjà les lignes de fracture que devront affronter leurs associations lors des prochaines négociations collectives: la scolarité, les générations, l'immigration. Parmi les limites, mentionnons que les traitements statistiques n'ont pas permis de faire la distinction entre les lieux de naissance des RSG immigrantes, alors qu'on peut faire l'hypothèse que les RSG originaires d'Europe de l'Ouest ou des États-Unis se trouvent dans une position différente des RSG nées en Europe de l'Est ou dans les pays du Sud. Également, nos résultats sousestiment certainement l'effet de l'appartenance ethnoculturelle des RSG de minorités visibles nées au Canada.

\section{RÉSUMÉ}

Le présent article porte sur une forme particulière de travail de soins, soit la garde d'enfants à domicile, telle qu'elle est exercée dans le cadre institutionnel québécois. Après avoir exposé la précarité des conditions d'emploi de celles qui exercent cette activité, formellement à titre de travailleuses indépendantes, il met l'accent sur l'appréciation subjective qu'elles en ont: quelle importance accordent-elles aux marges d'autonomie procurées par leur statut? Quelles voies privilégient-elles pour améliorer les conditions d'exercice de leur activité? Comment envisagentelles leur avenir professionnel? Les résultats issus d'une enquête réalisée en 2011 auprès de 3769 responsables de services de garde en milieu familial révèlent une importante diversité à cet égard, expliquée par l'imbrication des inégalités construites dans la trajectoire professionnelle, de la division du travail au sein de la famille et de l'expérience de la migration.

Mots clés: travail de soins, travail indépendant, travail précaire, intersectionnalité des rapports sociaux, action collective

\section{ABSTRACT}

This article examines a special form of caregiving, home daycare, as it is practised under the institutional framework of Québec. After describing the precarious working conditions of the women involved in this activity, formally as independent workers, the article focuses on the subjective appreciation these workers have of their work: what is the importance they give to the degree of autonomy provided by their status? In their eyes, what are the best means to improve their working conditions? How do they see their professional future? The findings of a survey of 3,769 family daycare service heads carried out in 2011 show a great diversity in this respect. This 
can be explained by the interweaving of built inequalities in the professional path, the division of labour within the family and the migration experience.

Key words: care work, independent work, precarious work, intersectionality of social relations, collective action

\section{RESUMEN}

El presente artículo trata acerca de una forma particular del trabajo asociado a los cuidados, como lo son las guarderías de niños a domicilio, tal como se ejerce en el marco institucional quebequense. Después de exponer la precariedad de las condiciones de empleo de quienes ejercen esta actividad formalmente a título de trabajadoras independientes, se hace énfasis en su apreciación subjetiva: ¿Qué importancia le dan ellas al margen de autonomía procurado por su estatus? ¿Qué caminos prevén para mejorar las condiciones de ejercicio de su actividad? ¿Cómo imaginan su futuro profesional? Los resultados de una encuesta realizada en 2011 a 3769 personas responsables de servicios de guardería en medio familiar, revelan una importante diversidad en este sentido, explicada por la implicación de las desigualdades construidas en la trayectoria profesional, la división del trabajo al interior de la familia y la experiencia migratoria.

Palabras clave: Trabajo en cuidados, trabajo independiente, trabajo precario, interseccionalidad de las relaciones sociales, acción colectiva

\section{BIBLIOGRAPHIE}

Akyeampong, E. B. et D. Sussman (2003), «Régimes de santé des travailleurs indépendants», L'emploi et le revenu en perspectives, mai, p. 15-21.

Aldrich, H. E. et R. Waldinger (1990), «Ethnicity and Entrepreneurship», Annual Review of Sociology, vol. 16, p. 111-135.

Apitzsch, U. et M. Kontos (dir.) (2003), «Self-employment, Gender and Migration», International Review of Sociology, vol. 13, n 1, p. 67-76.

Avril C. et M. Cartier (2014), «Subordination in Home Service Jobs: Comparing Providers of HomeBased Child Care, Elder Care, and Cleaning in France», Gender \& Society, vol. 28, n 4, p. 609-630.

AvriL, C. (2012), «Ressources et lignes de clivage parmi les aides à domicile. Spécifier une position sociale: quelles opérations de recherche?», Actes de la recherche en sciences sociales, n 191-192, p. 86-105.

AvriL, C. (2003), «Quel lien entre travail et classe sociale pour les travailleuses du bas de l'échelle? L'exemple des aides à domicile auprès des personnes âgées dépendantes», Lien social et Politiques, $\mathrm{n}^{\circ} 49, \mathrm{p} .147-154$.

Arum, R. (1997), «Trends in Male and Female Self-Employment: Growth in a New Middle Class or Increasing Marginalization of the Labor Force?», Research in Social Stratification and Mobility, vol. 15, p. 209-238.

BeAulieu, V. (2011), Les conditions de travail et la protection sociale des travailleurs vulnérables. Le cas des responsables d'un service de garde en milieu familial. Mémoire présenté à la faculté des études supérieures de l'Université Laval dans le cadre du programme de maîtrise en relations industrielles.

Bernstein, S. (2012), «Sector-based Collective Bargaining Regimes and Gender Segregation: A Case Study of Self-employed Home Childcare Workers", in Fudge, J., S. McCrystal et K. Sankaran (dir.), Challenging the Legal Boundaries of Work Regulation, Oxford et Portland Oregon, Onati International Series in Law and Society, p. 213-229.

BiLGE, S. (2010). «De l'analogie à l'articulation: théoriser la différenciation sociale et l'inégalité complexe », L'Homme et la société, vol. 2, nº 176-177, p. 43-64. 
Bisson, J. (2012), Identités professionnelles et représentation collective: le cas des responsables de services de garde en milieu familial (RSG), Mémoire présenté à la Faculté des études supérieures de l'Université Laval dans le cadre du programme de maîtrise en relations industrielles.

CARR, D. (1996), «Two paths to self-employment. Women's and men's self-employment in the United States, 1980 », Work \& Occupations, vol. 23, n 1, fév., p. 26-53.

Cartier, M., E. d'Halluin, M.-H. Lechien et J. Rousseau (2012). La «sous-activité» des assistantes maternelles: un rapport au métier différencié selon le positionnement social. Politiques sociales et familiales, $\mathrm{n}^{\circ} 109$, septembre, p. 35-46.

Cognet, M. (2010), «Genre et ethnicité dans la division du travail en santé: la responsabilité politique des États», L'Homme et la société, vol. 2 (n 176-177), p. 101-129.

Cox, R. (2005), Pour en faire un véritable emploi: des stratégies pour améliorer les conditions de travail des responsables de services de garde en milieu familial, Ottawa, Condition féminine Canada, $122 \mathrm{p}$.

D'Amours, M. (2015), «La relation d'emploi contemporaine: du rapport salarial à la relation sociale de travail», in D' Amours, M., S. A. Soussi et D.-G. Tremblay (dir.), Repenser le travail et ses mutations: des concepts nouveaux pour des réalités transformées, Presses de l'Université du Québec, p. 135-164.

D’Amours, M., avec la collaboration de J. Bisson (2013). Identités au travail et représentation collective. Le cas des responsables de services de garde en milieu familial (RSG). Rapport synthèse, Québec, Université Laval, ARUC travail et emploi, Cahier de transfert 2013-005, $64 \mathrm{p}$.

D'Amours, M. (2010). «Les logiques d'action collective d'associations regroupant des travailleurs indépendants", Relations industrielles/Industrial Relations, vol. 65, nº 2, juin, p. 257-279.

D’Amours, M. et S. Crespo (2004). «Les dimensions de l'hétérogénéité de la catégorie de travailleur indépendant sans employé: éléments pour une typologie», Relations industrielles/Industrial Relations, vol. 59, $\mathrm{n}^{\circ} 3$, p. 459-489.

Delage, B. (2002), Résultats de l'enquête sur le travail indépendant au Canada. Développement des Ressources humaines Canada, Direction générale de la recherche appliquée, SP-465-01-02F.

Devetter, F.-X. (2012), «Qualité de l'emploi et des conditions de travail des professionnels-le-s de la petite enfance», Politiques sociales et familiales, $\mathrm{n}^{\circ}$ 109, septembre, p. 9-22.

GOUVERNEMENT DU QUÉBEC (2015), Situation des centres de la petite enfance, des garderies et de la garde en milieu familial au Québec en 2013. Québec, mars.

GOUVERNEMENT DU QUÉBEC (2014), Situation des centres de la petite enfance, des garderies et de la garde en milieu familial au Québec, 2012. Québec, janvier.

Hosmer, D. W., S. Lemeshow et R. X. Sturdivant (2013), Applied Logistic Regression, New York, Wiley and Sons Inc.

Inkson, K. et M. B. Arthur (2001), «How to Be a Successful Career Capitalist», Organizational Dynamics, vol. $30, n^{\circ} 1$, p. $48-61$,

JurIK, N. C. (1998), «Getting Away and Getting By: the Experiences of Self-Employed Homeworkers», Work and Occupations, vol. 25, $\mathrm{n}^{\circ} 1$, février, p. 7-35.

Kergoat, D. (2009), «Dynamique et consubstantialité des rapports sociaux», dans E. Dorlin (dir.), Sexe, race, classe, pour une épistémologie de la domination, Paris, PUF, p. 111-125.

Lalonde-Graton, M. (2002), Des salles d'asile aux centres de la petite enfance: La petite histoire des services de garde au Québec, Québec, Presse de l'Université du Québec.

Marler, J. H., M. W. Barringer et G. T. Milkovich (2002), «Boundaryless and traditional contingent employees: worlds apart», Journal of Organizational Behavior, n 23, p. 425-453.

Mахім, P. S. (1992), «Immigrants, Visible Minorities, and Self-Employment», Demography, vol. 29, n² 2, mai, p. 181-198.

NaKano Glenn, E. (2009), De la servitude au travail de service: les continuités historiques de la division raciale du travail reproductif payé», dans Dorlin, E. (dir.), Sexe, race, classe. Pour une épistémologie de la domination, Paris, PUF, coll. Actuel/Marx confrontation, p. 21-63.

Palomares E. et A. Testenoire (2010), «Indissociables et irréductibles: les rapports sociaux de genre, ethniques et de classe», L'Homme et la société, nº 176-177, p. 15-26. 
PeEL, S. et K. InKson (2004), «Contracting and careers: choosing between self and organizational employement», Career Development International, vol. 9, n 6, p. 542-558.

TANG, J. (1995), «Differences in the Process of Self-employment among Whites, Blacks, and Asians: The Case of Scientists and Engineers», Sociological Perspectives, vol. 38, n 2, p. 273-309.

Vosko L. F. et N. Zuкewich (2006), «Precarious by Choice? Gender and Self- Employment», in Vosko, L. F. (dir.), Precarious Employment: Understanding Labour Market Insecurity in Canada. Montreal and Kingston, McGill-Queen's University Press.

Vosкo, L. F. (2006), Precarious Employment: Understanding Labour Market Insecurity in Canada, Montreal and Kingston, McGill-Queen's University Press

Zavella, P. (1997), «Constructing Ethnic Identity with "Chicana” Informants», in Lamphere, L., H. Ragoné et P. Zavella (dir.), Situates Lives. Gender and Culture in Everyday Life, New York, Routledge. 